\title{
Finite Element Approximation of the Viscoelastic Flow Problem: A non-Residual Based Stabilized Formulation
}

\author{
E. Castillo ${ }^{\mathrm{a}, \mathrm{b}}$ and R. Codina $\mathrm{a}^{\mathrm{b}, \mathrm{c}}$ \\ ${ }^{a}$ Universitat Politècnica de Catalunya, Jordi Girona 1-3, Edifici C1, 08034 Barcelona, Spain \\ ${ }^{\mathrm{b}}$ Universidad de Santiago de Chile (USACH), Depto. de Ingeniería Mecánica, Av. Bdo. O’Higgins 3363, Santiago, Chile \\ ${ }^{\mathrm{c}}$ CIMNE International Center for Numerical Methods in Engineering, Gran Capità S/N, 08034 Barcelona, Spain
}

\begin{abstract}
In this paper, a three-field finite element stabilized formulation for the incompressible viscoelastic fluid flow problem is tested numerically. Starting from a residual based formulation, a non-residual based one is designed, the benefits of which are highlighted in this work. Both formulations allow one to deal with the convective nature of the problem and to use equal interpolation for the problem unknowns $\boldsymbol{\sigma}-\boldsymbol{u}-\boldsymbol{p}$ (deviatoric stress, velocity and pressure). Additionally, some results from the numerical analysis of the formulation are stated. Numerical examples are presented to show the robustness of the method, which include the classical $4: 1$ planar contraction problem and the flow over a confined cylinder case, as well as a two-fluid formulation for the planar jet buckling problem.
\end{abstract}

Keywords:

Viscoelastic fluids, Oldroyd-B fluids, Stabilized finite element methods, Variational Multiscale.

\section{Introduction}

The finite element approximation of the flow of viscoelastic fluids presents several numerical difficulties, particularly for the spatial approximation. On the one hand, the finite element approximation used must satisfy two compatibility or inf-sup conditions, the first between velocity-pressure and the second associated to the interpolation of velocity and stress $[1,2]$. On the other hand, the convective nature presented both in the momentum and constitutive equation may lead to numerical instabilities.

The advective nature of the constitutive equation makes it necessary to use a stabilized formulation to avoid global oscillations. In the context of the finite element method, many algorithms have been developed to solve this problem: the classical SUPG method and its non-consistent counterpart, the SU method [3], the GLS method [4,5], and the stabilization based on the discontinuous Galerkin method [6]. In the present work, we apply two stabilized formulations based on the Variational Multiscale (VMS) framework introduced

Email address: ernesto.castillode@usach.cl and ramon.codina@upc.edu (E. Castillo ${ }^{\mathrm{a}, \mathrm{b}}$ and R. Codina $^{\mathrm{b}, \mathrm{c}}$ ) 
by Hughes et al. [7] for the scalar convection-diffusion-reaction problem, and extended later to the vectorial Stokes problem in [8], where the space of the sub-grid scales is taken orthogonal to the finite element space. As we shall see, this is an important ingredient in the design of our formulations.

The starting point of a VMS approach is to split the unknowns of the problem into two components, namely, the component that can be approximated by the finite element mesh and the unresolvable one, called sub-grid scale or simply sub-scale in what follows. The latter needs to be approximated in a simple manner in terms of the former, so as to capture its main effect and yield a stable formulation for the finite element unknown. There are different ways to approximate the sub-scale and, in particular, to choose the space where it is taken. We will describe two formulations which precisely differ in this choice. Both formulations will allow one to deal with the instabilities of the three-field viscoelastic formulation described earlier. There will be no need to meet the inf-sup conditions for the interpolation spaces and it will be possible to solve convection dominated problems both in the momentum and in the constitutive equation. For the latter, these methods have been found to work well. However, for the momentum equation we have observed that they are not robust in the presence of high gradients of the unknowns, and therefore we have had to modify them. The modification consists in designing a sort of term-by-term stabilization based on the choice of subscales orthogonal to the finite element space. We will describe in detail this method and the need for it.

The work is organized as follows. Section 2 contains the presentation of the problem. Section 3 presents our stabilized finite element approach. Section 4 is devoted to the numerical analysis results and Section 5 contains typical numerical examples used for this kind of fluids. Finally, in Section 6 conclusions are summarized.

\section{The viscoelastic flow problem}

\subsection{Boundary value problem}

To simulate the transitory incompressible and isothermal flow of viscoelastic fluids, one needs solve the momentum balance equation, the continuity equation and a constitutive equation that defines the viscoelastic contribution of the fluid.

The conservation equations for momentum and mass may be expressed as:

$$
\begin{aligned}
\rho \frac{\partial \boldsymbol{u}}{\partial t}+\rho \boldsymbol{u} \cdot \nabla \boldsymbol{u}-\nabla \cdot \boldsymbol{T}+\nabla p=\boldsymbol{f} & \text { in } \Omega, t \in] 0, t_{f}[ \\
\nabla \cdot \boldsymbol{u}=0 & \text { in } \Omega, t \in] 0, t_{f}[
\end{aligned}
$$

where $\Omega$ is the computational domain of $\mathbb{R}^{d}$ occupied by the fluid, $] 0, t_{f}[$ is the time interval where the problem is solved, $\rho$ denotes the constant density, $p: \Omega \rightarrow \mathbb{R}$ the pressure, $\boldsymbol{u}: \Omega \rightarrow \mathbb{R}^{d}$ the velocity vector, $f: \Omega \rightarrow \mathbb{R}^{d}$ the force vector and $\boldsymbol{T}: \Omega \rightarrow \mathbb{R}^{d} \otimes \mathbb{R}^{d}$ the deviatoric extra stress tensor which can be defined in 
terms of a viscous and a viscoelastic or elastic contribution as:

$$
\boldsymbol{T}=2 \beta \eta_{0} \nabla^{s} \boldsymbol{u}+\boldsymbol{\sigma}
$$

where $\beta \in[0,1]$ is a real parameter (ratio) to define the amount of viscous or solvent viscosity $\beta \eta_{0}$ and elastic or polymeric viscosity $(1-\beta) \eta_{0}$ in the fluid. For viscoelastic fluids, the problem is incomplete without the definition of a constitutive equation for the elastic part of the extra stress tensor $(\boldsymbol{\sigma})$. A large variety of approaches exist to define it (see $[9,10]$ for a complete description). In this work, only the differential Oldroyd-B model is considered. It reads:

$$
\left.\frac{\lambda}{2 \eta_{0}} \frac{\partial \boldsymbol{\sigma}}{\partial t}+\frac{1}{2 \eta_{0}} \boldsymbol{\sigma}-(1-\beta) \nabla^{s} \boldsymbol{u}+\frac{\lambda}{2 \eta_{0}}\left(\boldsymbol{u} \cdot \nabla \boldsymbol{\sigma}-\boldsymbol{\sigma} \cdot \nabla \boldsymbol{u}-(\nabla \boldsymbol{u})^{T} \cdot \boldsymbol{\sigma}\right)=\mathbf{0} \quad \text { in } \Omega, t \in\right] 0, t_{f}[
$$

where $\lambda$ is the relaxation time.

Calling $\boldsymbol{U}=[\boldsymbol{u}, \boldsymbol{\sigma}, p], \boldsymbol{F}=[\boldsymbol{f}, 0,0]$ and defining

$$
\mathcal{L}(\hat{\boldsymbol{u}}, \boldsymbol{U}):=\left(\begin{array}{c}
\rho \hat{\boldsymbol{u}} \cdot \nabla \boldsymbol{u}-2 \beta \eta_{0} \nabla \cdot\left(\nabla^{s} \boldsymbol{u}\right)-\nabla \cdot \boldsymbol{\sigma}+\nabla p \\
\nabla \cdot \boldsymbol{u} \\
\frac{\lambda}{2 \eta_{0}} \frac{\partial \boldsymbol{\sigma}}{\partial t}+\frac{1}{2 \eta_{0}} \boldsymbol{\sigma}-(1-\beta) \nabla^{s} \boldsymbol{u}+\frac{\lambda}{2 \eta_{0}}\left(\hat{\boldsymbol{u}} \cdot \nabla \boldsymbol{\sigma}-\boldsymbol{\sigma} \cdot \nabla \hat{\boldsymbol{u}}-(\nabla \hat{\boldsymbol{u}})^{T} \cdot \boldsymbol{\sigma}\right)
\end{array}\right)
$$

and

$$
T(\boldsymbol{U}):=\left(\begin{array}{c}
\rho \frac{\partial \boldsymbol{u}}{\partial t} \\
0 \\
\frac{\lambda}{2 \eta_{0}} \frac{\partial \boldsymbol{\sigma}}{\partial t}
\end{array}\right)
$$

we may write (1), (2) and (4) using the definition (3) as $T(\boldsymbol{U})+\mathcal{L}(\hat{\boldsymbol{u}}, \boldsymbol{U})=\boldsymbol{F}$.

Initial and boundary conditions have to be appended to problem (1)-(4). For simplicity in the exposition, we will consider the simplest boundary condition $\boldsymbol{u}=\mathbf{0}$ on $\partial \Omega$, and no boundary conditions for $\boldsymbol{\sigma}$. However, due to the hyperbolic nature of the constitutive equation, in some examples the elastic stresses are fixed on the inflow part of the boundary. For a complete description of the mathematical structure of the problem we refer to [11].

\subsection{The variational form}

We introduce some notation in order to write the weak form of the problem. The space of square integrable functions in a domain $\omega$ is denoted by $L^{2}(\omega)$, and the space of functions whose distributional derivatives of order up to $m \geq 0$ (integer) belong to $L^{2}(\omega)$ by $H^{m}(\omega)$. The space $H_{0}^{m}(\omega)$ consists of functions in $H^{1}(\omega)$ vanishing on $\partial \omega$. The topological dual of $H_{0}^{1}(\Omega)$ is denoted by $H^{-1}(\Omega)$, the duality pairing by $\langle\cdot, \cdot\rangle$, and the $L^{2}$ inner product in $\omega$ (for scalar, vectors and tensors) is denoted by $(\cdot, \cdot)$. The norm in a functional space $X$ 
will be denoted by $\|\cdot\|_{X}$, except when $X=L^{2}(\Omega)$, case in which the subscript will be omitted.

Using this notation, the stress, velocity and pressure functional spaces for the continuous problem are $\Upsilon_{0}=L^{2}\left(0, t_{f} ;\left(H_{0}^{1}(\Omega)\right)_{\text {sym }}^{d \times d}\right)$ (the subscript sym standing for symmetric tensors), $\mathcal{V}_{0}=L^{2}\left(0, t_{f} ;\left(H_{0}^{1}(\Omega)\right)^{d}\right)$ and $\mathcal{Q}=\mathcal{D}\left(0, t_{f} ;\left(L^{2}(\Omega) / \mathbb{R}\right)\right)$ ( $\mathcal{D}$ standing for distributions), respectively. The weak form of the problem consists in finding $\boldsymbol{U}=[\boldsymbol{u}, p, \boldsymbol{\sigma}] \in \mathcal{X}=\mathcal{V}_{0} \times \mathcal{Q} \times \boldsymbol{\Upsilon}_{0}$, such that:

$$
\begin{aligned}
\left(\rho \frac{\partial \boldsymbol{u}}{\partial t}, \boldsymbol{v}\right)+2\left(\beta \eta_{0} \nabla^{s} \boldsymbol{u}, \nabla^{s} \boldsymbol{v}\right)+\langle\rho \boldsymbol{u} \cdot \nabla \boldsymbol{u}, \boldsymbol{v}\rangle+\left(\boldsymbol{\sigma}, \nabla^{s} \boldsymbol{v}\right)-(p, \nabla \cdot \boldsymbol{v}) & =\langle\boldsymbol{f}, \boldsymbol{v}\rangle \\
(q, \nabla \cdot \boldsymbol{u}) & =0 \\
\left(\frac{\lambda}{2 \eta_{0}} \frac{\partial \boldsymbol{\sigma}}{\partial t}, \boldsymbol{\tau}\right)+\left(\frac{1}{2 \eta_{0}} \boldsymbol{\sigma}, \boldsymbol{\tau}\right)-\left((1-\beta) \nabla^{s} \boldsymbol{u}, \boldsymbol{\tau}\right)+\frac{\lambda}{2 \eta_{0}}\left(\boldsymbol{u} \cdot \nabla \boldsymbol{\sigma}-\boldsymbol{\sigma} \cdot \nabla \boldsymbol{u}-(\nabla \boldsymbol{u})^{T} \cdot \boldsymbol{\sigma}, \boldsymbol{\tau}\right) & =0
\end{aligned}
$$

for all $\boldsymbol{V}=[\boldsymbol{v}, q, \boldsymbol{\tau}] \in \hat{\mathcal{X}}$ (the time independent counterpart of $\mathcal{X})$, where $\boldsymbol{f} \in\left(0, t_{f} ;\left(H^{-1}(\Omega)\right)\right)$.

In a compact form, the problem (7)-(9) can be written as:

$$
\left(\rho \frac{\partial \boldsymbol{u}}{\partial t}, \boldsymbol{v}\right)+\left(\frac{\lambda}{2 \eta_{0}} \frac{\partial \boldsymbol{\sigma}}{\partial t}, \boldsymbol{\tau}\right)+B(\boldsymbol{u} ; \boldsymbol{U}, \boldsymbol{V})=\langle\boldsymbol{f}, \boldsymbol{v}\rangle
$$

for all $\boldsymbol{V} \in \hat{\mathcal{X}}$, where

$$
\begin{aligned}
B(\hat{\boldsymbol{u}} ; \boldsymbol{U}, \boldsymbol{V}) & =2\left(\beta \eta_{0} \nabla^{s} \boldsymbol{u}, \nabla^{s} \boldsymbol{v}\right)+\langle\rho \hat{\boldsymbol{u}} \cdot \nabla \boldsymbol{u}, \boldsymbol{v}\rangle+\left(\boldsymbol{\sigma}, \nabla^{s} \boldsymbol{v}\right)-(p, \nabla \cdot \boldsymbol{v})+(q, \nabla \cdot \boldsymbol{u}) \\
& +\left(\frac{1}{2 \eta_{0}} \boldsymbol{\sigma}, \boldsymbol{\tau}\right)-\left((1-\beta) \nabla^{s} \boldsymbol{u}, \boldsymbol{\tau}\right)+\frac{\lambda}{2 \eta_{0}}\left(\hat{\boldsymbol{u}} \cdot \nabla \boldsymbol{\sigma}-\boldsymbol{\sigma} \cdot \nabla \hat{\boldsymbol{u}}-(\nabla \hat{\boldsymbol{u}})^{T} \cdot \boldsymbol{\sigma}, \boldsymbol{\tau}\right)
\end{aligned}
$$

\section{Numerical approximation}

\subsection{Galerkin finite element discretization}

The standard Galerkin approximation for the variational problem can be performed by considering a finite element partition $\mathcal{T}_{h}$ of the domain $\Omega$. The diameter of an element domain $K \in \mathcal{T}_{h}$ is denoted by $h_{K}$ and the diameter of the element partition is defined by $h=\max \left\{h_{K} \mid K \in \mathcal{T}_{h}\right\}$. Under the above considerations, we can construct conforming finite elements spaces, $\mathcal{V}_{h, 0} \subset \mathcal{V}_{0}, \mathcal{Q}_{h} \subset \mathcal{Q}$ and $\boldsymbol{\Upsilon}_{h, 0} \subset \boldsymbol{\Upsilon}_{0}$ in the usual manner. If $\boldsymbol{\mathcal { X }}_{h}=\mathcal{V}_{h, 0} \times \mathcal{Q}_{h} \times \boldsymbol{\Upsilon}_{h, 0}$, and $\boldsymbol{U}_{h}=\left[\boldsymbol{u}_{h}, p_{h}, \boldsymbol{\sigma}_{h}\right]$, the Galerkin finite element approximation consist in finding $\boldsymbol{U}_{h} \in \mathcal{X}_{h}$, such that:

$$
\left(\rho \frac{\partial \boldsymbol{u}_{h}}{\partial t}, \boldsymbol{v}_{h}\right)+\left(\frac{\lambda}{2 \eta_{0}} \frac{\partial \boldsymbol{\sigma}_{h}}{\partial t}, \boldsymbol{\tau}_{h}\right)+B\left(\boldsymbol{u}_{h} ; \boldsymbol{U}_{h}, \boldsymbol{V}_{h}\right)=\left\langle\boldsymbol{f}, \boldsymbol{v}_{h}\right\rangle
$$

for all $\boldsymbol{V}_{h}=\left[\boldsymbol{v}_{h}, q_{h}, \boldsymbol{\tau}_{h}\right] \in \hat{\mathcal{X}}_{h}$.

In principle, we have posed no restrictions on the choice of the finite element spaces. However, there are restrictions that must be satisfied explicitly in the discrete formulation used. These are the same as for 
the three-field formulation of the Stokes problem [12, 1, 13]. A possible remedy to this situation is to use inf-sup stable elements (see [3] for the $2 D$ case and [14] for the $3 D$ case). The other possibility is to use a stabilized formulation permitting any interpolation between the variables, which is the approach used in this work. In general, a stabilized formulation consists of replacing $B$ by another bilinear form $B_{h}$, possibly mesh dependent, with better stabilities properties.

\subsection{Stabilized finite element approximation}

In the following we present two stabilized finite element formulations for computing viscoelastic flows using the Oldroyd-B constitutive model. They are based on the variational multi-scale approach, where the unknown can be split as $\boldsymbol{U}=\boldsymbol{U}_{h}+\tilde{\boldsymbol{U}}$, where $\boldsymbol{U}_{h}$ is the finite element part and $\tilde{\boldsymbol{U}}$ represents the remainder non-captured part, called the sub-grid scale. A similar split can be considered for the test functions. The first method to be discussed is a residual based stabilized formulation, whereas the second is a non-residual one which turns out to have a better performance in situations in which the solution is not smooth.

\subsubsection{Residual based stabilized finite element formulation}

After some approximations (see [15]), the residual based method we consider consists in finding $\boldsymbol{U}_{h} \in \boldsymbol{\mathcal { X }}_{h}$ such that

$$
\left(\rho \frac{\partial \boldsymbol{u}_{h}}{\partial t}, \boldsymbol{v}_{h}\right)+\left(\frac{\lambda}{2 \eta_{0}} \frac{\partial \boldsymbol{\sigma}_{h}}{\partial t}, \boldsymbol{\tau}_{h}\right)+B\left(\boldsymbol{u}_{h} ; \boldsymbol{U}_{h}, \boldsymbol{V}_{h}\right)+\sum_{K}\left\langle\tilde{\boldsymbol{U}}, \mathcal{L}^{*}\left(\boldsymbol{u}_{h} ; \boldsymbol{V}_{h}\right)\right\rangle_{K}=\left\langle\boldsymbol{f}, \boldsymbol{v}_{h}\right\rangle
$$

for all $\boldsymbol{V}_{h} \in \hat{\boldsymbol{\mathcal { X }}}_{h}$, where $\mathcal{L}^{*}\left(\hat{\boldsymbol{u}}, \boldsymbol{V}_{h}\right)$ is the formal adjoint of the operator in (5) without considering boundary conditions, and $\tilde{\boldsymbol{U}}$ is the subgrid scale, which needs to be approximated. If $\tilde{P}$ is the $L^{2}$ projection onto the space of sub-grid scales, the approximation we consider within each element is

$$
\tilde{\boldsymbol{U}}=\boldsymbol{\alpha} \tilde{P}\left[\boldsymbol{R}_{h}\right]=\boldsymbol{\alpha} \tilde{P}\left[\boldsymbol{F}-\mathcal{L}\left(\boldsymbol{u}_{h} ; \boldsymbol{U}_{h}\right)\right]
$$

where $\boldsymbol{R}_{h}$ is the finite element residual and $\boldsymbol{\alpha}$ is a matrix computed within each element, which we take as

$$
\boldsymbol{\alpha}=\operatorname{diag}\left(\alpha_{1} \boldsymbol{I}_{d}, \alpha_{2}, \alpha_{3} \boldsymbol{I}_{d \times d}\right)
$$

with $\boldsymbol{I}_{d}$ the identity on vectors of $\mathbb{R}^{d}, \boldsymbol{I}_{d \times d}$ the identity on second order tensors and the parameters $\alpha_{i}$, $i=1,2,3$, are computed as

$$
\alpha_{1}=\left[c_{1} \frac{\eta_{0}}{h^{2}}+c_{2} \frac{\rho\left|\boldsymbol{u}_{h}\right|}{h}\right]^{-1}, \quad \alpha_{2}=\frac{h^{2}}{c_{1} \alpha_{1}}, \quad \alpha_{3}=\left[c_{1} \frac{1}{2 \eta_{0}}+c_{3} \frac{\lambda}{\eta_{0}}\left(\frac{\left|\boldsymbol{u}_{h}\right|}{2 h}+\left|\nabla \boldsymbol{u}_{h}\right|\right)\right]^{-1}
$$

where the $c_{1}, c_{2}$ and $c_{3}$ are algorithmic constants. 
Independently of the choice of the projection $\tilde{P}$, the method defined by (12) is consistent, since the terms added to the Galerkin ones are proportional to the finite element residual $\boldsymbol{R}_{h}$. Thus, they vanish if the finite element solution is replaced by the continuous solution $\boldsymbol{U}$. It is in this sense that we call (12) a residual based method.

\subsubsection{Non-Residual based stabilized finite element formulation}

For smooth solution, method (12) is stable and displays optimal order of convergence. Considering $\tilde{P}=P_{h}^{\perp}$, from (12) we can design a simplified method, which consist in neglecting the cross local innerproduct terms, as well as some other terms that do not contribute to stability. In particular, from the momentum equation we can obtain the following stabilization term

$$
\begin{aligned}
S_{1}^{\perp}\left(\hat{\boldsymbol{u}}_{h} ; \boldsymbol{U}_{h}, \boldsymbol{V}_{h}\right)= & \sum_{K} \alpha_{1}\left\langle P_{h}^{\perp}\left[\rho \hat{\boldsymbol{u}}_{h} \cdot \nabla \boldsymbol{u}_{h}\right], \rho \hat{\boldsymbol{u}}_{h} \cdot \nabla \boldsymbol{v}_{h}\right\rangle_{K}+\sum_{K} \alpha_{1}\left\langle P_{h}^{\perp}\left[\nabla p_{h}\right], \nabla q_{h}\right\rangle_{K} \\
& +(1-\beta) \sum_{K} \alpha_{1}\left\langle P_{h}^{\perp}\left[\nabla \cdot \boldsymbol{\sigma}_{h}\right], \nabla \cdot \boldsymbol{\tau}_{h}\right\rangle_{K}
\end{aligned}
$$

instead of the following two terms derived from the residual based method:

$$
\begin{aligned}
\tilde{S}_{1}\left(\hat{\boldsymbol{u}}_{h} ; \boldsymbol{U}_{h}, \boldsymbol{V}_{h}\right)= & \sum_{K} \alpha_{1}\left\langle\tilde{P}\left[-\nabla \cdot \boldsymbol{\sigma}_{h}-2 \beta \eta_{0} \nabla \cdot\left(\nabla^{s} \boldsymbol{u}_{h}\right)+\rho \hat{\boldsymbol{u}}_{h} \cdot \nabla \boldsymbol{u}_{h}+\nabla p_{h}\right],\right. \\
& \left.-(1-\beta) \nabla \cdot \boldsymbol{\tau}_{h}+2 \beta \eta_{0} \nabla \cdot\left(\nabla^{s} \boldsymbol{v}_{h}\right)+\rho \hat{\boldsymbol{u}}_{h} \cdot \nabla \boldsymbol{v}_{h}+\nabla q_{h}\right\rangle_{K}
\end{aligned}
$$

that would contribute to the left-hand-side of the discrete problem and

$$
\tilde{R}_{1}\left(\hat{\boldsymbol{u}}_{h} ; \boldsymbol{V}_{h}\right)=\sum_{K} \alpha_{1}\left\langle\tilde{P}[\boldsymbol{f}],-(1-\beta) \nabla \cdot \boldsymbol{\tau}_{h}+2 \beta \eta_{0} \nabla \cdot\left(\nabla^{s} \boldsymbol{v}_{h}\right)+\rho \hat{\boldsymbol{u}}_{h} \cdot \nabla \boldsymbol{v}_{h}+\nabla q_{h}\right\rangle_{K}
$$

that would appear in the right-hand-side of the discrete problem.

Note that the use of (13) intead of (14) and (15) is not just a simplification. For smooth solutions, both methods have optimal convergence rate in $h$. However, in problems where the solution has strong gradients, we found (13) more robust. When using (13), the terms that have identified as "problematic" are the cross-terms $\alpha_{1}\left\langle\nabla p_{h},-(1-\beta) \nabla \cdot \boldsymbol{\tau}_{h}\right\rangle_{K}$ and $\alpha_{1}\left\langle-\nabla \cdot \boldsymbol{\sigma}_{h}, \nabla q_{h}\right\rangle_{K}$. In the presence of high derivatives of pressure and stress, such as in the corner 4:1 contraction example, they lead to convergence difficulties for high Weissenberg numbers and to inaccurate localization of pressure and stress peaks. This is a fact that we have confirmed from several numerical experiments.

For the stabilization terms of the constitutive equation and the continuity equation we may still use the residual based form, which has proven to be a very robust combination with the additional advantage of a smaller number of finite element projections needed to construct the method. For the continuity and 
constitutive equation the stabilization terms are defined respectively as:

$$
\begin{aligned}
\tilde{S}_{2}\left(\boldsymbol{U}_{h}, \boldsymbol{V}_{h}\right)= & \sum_{K} \alpha_{3}\left\langle\tilde{P}\left[\nabla \cdot \boldsymbol{u}_{h}\right], \nabla \cdot \boldsymbol{v}_{h}\right\rangle_{K}, \\
\tilde{S}_{3}\left(\hat{\boldsymbol{u}}_{h} ; \boldsymbol{U}_{h}, \boldsymbol{V}_{h}\right)= & \sum_{K} \alpha_{3}\left\langle\tilde{P}\left[\frac{1}{2 \eta_{0}} \boldsymbol{\sigma}_{h}-(1-\beta) \nabla^{s} \boldsymbol{u}_{h}+\frac{\lambda}{2 \eta_{0}}\left(\hat{\boldsymbol{u}}_{h} \cdot \nabla \boldsymbol{\sigma}_{h}-\boldsymbol{\sigma}_{h} \cdot \nabla \hat{\boldsymbol{u}}_{h}-\left(\nabla \hat{\boldsymbol{u}}_{h}\right)^{T} \cdot \boldsymbol{\sigma}_{h}\right)\right]\right. \\
& \left.-\frac{1}{2 \eta_{0}} \boldsymbol{\tau}_{h}-\nabla^{s} \boldsymbol{v}_{h}+\frac{\lambda}{2 \eta_{0}}\left(\hat{\boldsymbol{u}}_{h} \cdot \nabla \boldsymbol{\tau}_{h}+\boldsymbol{\tau}_{h} \cdot\left(\nabla \hat{\boldsymbol{u}}_{h}\right)^{T}+\nabla \hat{\boldsymbol{u}}_{h} \cdot \boldsymbol{\tau}_{h}\right)\right\rangle_{K}
\end{aligned}
$$

Finally, the proposed method can be defined as follows: find $\boldsymbol{U}_{h} \in \boldsymbol{\mathcal { X }}_{h}$ such that

$$
\begin{aligned}
& \left(\rho \frac{\partial \boldsymbol{u}_{h}}{\partial t}, \boldsymbol{v}_{h}\right)+\left(\frac{\lambda}{2 \eta_{0}} \frac{\partial \boldsymbol{\sigma}_{h}}{\partial t}, \boldsymbol{\tau}_{h}\right)+B\left(\boldsymbol{u} ; \boldsymbol{U}_{h}, \boldsymbol{V}_{h}\right) \\
& \quad+S_{1}^{\perp}\left(\hat{\boldsymbol{u}}_{h} ; \boldsymbol{U}_{h}, \boldsymbol{V}_{h}\right)+\tilde{S}_{2}\left(\boldsymbol{U}_{h}, \boldsymbol{V}_{h}\right)+\tilde{S}_{3}\left(\hat{\boldsymbol{u}}_{h} ; \boldsymbol{U}_{h}, \boldsymbol{V}_{h}\right)=\left\langle\boldsymbol{f}, \boldsymbol{v}_{h}\right\rangle
\end{aligned}
$$

for all $\boldsymbol{V}_{h} \in \hat{\mathcal{X}}_{h}$. This replaces the residual formulation defined in (12). In the numerical examples and for the following analysis results, we use $\tilde{P}=P_{h}^{\perp}$ in the terms $\tilde{S}_{2}$ and $\tilde{S}_{3}$.

Both methods, the residual and the non-residual, are optimally convergent in space. The temporal order accuracy in the monolithic case is determined by the time integration scheme used. In the case of a fractional step approach, different algorithms could be designed. See [16], where first, second third order schemes in time were designed for the three-field viscoelastic case.

\section{Numerical Analysis Results}

We have performed a complete numerical analysis of the stationary and linearized problem, proving stability and convergence in the mesh dependent norm associated to the problem and in natural norms. We present here the results of this analysis without proof, which can be found in [17].

The problem we consider is the following: find $\boldsymbol{U}_{h} \in \boldsymbol{\mathcal { X }}_{h}$ (now a space of time independent functions) such that

$$
B_{\text {stab }}\left(\boldsymbol{U}_{h}, \boldsymbol{V}_{h}\right):=B\left(\boldsymbol{a}_{h} ; \boldsymbol{U}_{h}, \boldsymbol{V}_{h}\right)+\sum_{K}\left\langle\tilde{\boldsymbol{U}}, \mathcal{L}^{*}\left(\boldsymbol{a}_{h} ; \boldsymbol{V}_{h}\right)\right\rangle_{K}=\left\langle\boldsymbol{f}, \boldsymbol{v}_{h}\right\rangle
$$

for all $\boldsymbol{V}_{h} \in \mathcal{X}_{h}$, with the adequate approximation for $\tilde{\boldsymbol{U}}$. Here, $\boldsymbol{a}_{h}$ is a given advection velocity, which in a fixed-point like iterative algorithm can be understood as the velocity field obtained in a previous iteration. To guarantee the well-posedness in the solution of the problem (19), the advection velocity must satisfy the following assumption (see e.g. [11]):

$$
\boldsymbol{a}_{h} \in \mathcal{V}_{0}, \nabla \cdot \boldsymbol{a}_{h}=0,\left\|\boldsymbol{a}_{h}\right\|_{\infty} \leq M_{a},\left\|\nabla \boldsymbol{a}_{h}\right\|_{\infty} \leq M_{g} \text { for some constants } M_{a}, M_{g}<\infty
$$


Let us assume for simplicity that the stabilization parameters are constant. The norm associated to the stabilized bilinear form $B_{\text {stab }}$, in which the results will be first presented, is given by

$$
\begin{aligned}
\left\|\boldsymbol{V}_{h}\right\|_{W}^{2} & =2 \beta \mu(1-\beta)\left\|\nabla^{s} \boldsymbol{v}_{h}\right\|^{2}+\frac{1}{2 \mu}\left\|\boldsymbol{\tau}_{h}\right\|^{2}+\alpha_{1}\left\|\rho \boldsymbol{a}_{h} \cdot \nabla \boldsymbol{v}_{h}+\nabla q_{h}-\nabla \cdot \boldsymbol{\tau}_{h}\right\|^{2} \\
& +\alpha_{1}\left\|P_{u}^{\perp}\left(\rho \boldsymbol{a}_{h} \cdot \nabla \boldsymbol{v}_{h}\right)\right\|^{2}+\alpha_{1}(1-\beta) \sum_{K}\left\|P_{u}^{\perp}\left(\nabla \cdot \boldsymbol{\tau}_{h}\right)\right\|^{2}+\alpha_{1} \sum_{K}\left\|P_{u}^{\perp}\left(\nabla q_{h}\right)\right\|^{2} \\
& +\alpha_{2}\left\|\nabla \cdot \boldsymbol{v}_{h}\right\|^{2}+\alpha_{3}\left\|-(1-\beta) \nabla^{s} \boldsymbol{v}_{h}+\frac{\lambda}{2 \mu}\left(\boldsymbol{a}_{h} \cdot \nabla \boldsymbol{\tau}_{h}+\dot{\boldsymbol{\tau}}_{h}^{* *}\right)\right\|^{2}
\end{aligned}
$$

where $\dot{\boldsymbol{\tau}}_{h}^{* *}=\boldsymbol{\tau}_{h} \cdot \nabla^{\mathrm{as}} \boldsymbol{a}_{h}-\nabla^{\mathrm{as}} \boldsymbol{a}_{h} \cdot \boldsymbol{\sigma}_{h}$ and $\nabla^{\text {as }}$ stands for the skew-symmetric part of the gradient of a vector field.

The stability result we have is the following:

Theorem 1 (stability). There is a constant $C>0$, such that

$$
\inf _{\boldsymbol{U}_{h} \in \mathcal{X}_{h} \boldsymbol{U}_{h} \in \mathcal{X}_{h}} \frac{B_{\text {stab }}\left(\boldsymbol{U}_{h}, \boldsymbol{V}_{h}\right)}{\left\|\boldsymbol{U}_{h}\right\|_{W}\left\|\boldsymbol{V}_{h}\right\|_{W}} \geq C
$$

Here and bellow, $C$ denotes a generic positive constant.

After having stability, we may proceed to prove convergence. The first ingredient is consistency. The method we have presented is not exactly consistent, but has an optimal consistency error. To state it, we need some additional notation. Let $\mathcal{W}_{h}$ be a finite element space of degree $k_{v}$. For any function $v \in H^{k_{v}^{\prime}+1}(\Omega)$ and for $i=0,1$, we define the interpolation errors $\varepsilon_{i}(v)$ from the interpolation estimates

$$
\inf _{v_{h} \in \mathcal{W}_{h}} \sum_{K}\left\|v-v_{h}\right\|_{H^{i}(K)} \leq C h^{k_{v}^{\prime \prime}+1-i} \sum_{K}\|v\|_{H^{k_{v}^{\prime \prime}+1}(K)}=: \varepsilon_{i}(v)
$$

where $k_{v}^{\prime \prime}=\min \left(k_{v}, k_{v}^{\prime}\right)$. We will denote by $\tilde{v}_{h}$ the best approximation of $v$ in $\mathcal{W}_{h}$. Clearly, we have that $\varepsilon_{0}(v)=h \varepsilon_{1}(v)$. This notation will allow us to prove that the error function of the method is

$$
E(h):=\sqrt{\mu} \varepsilon_{1}(\boldsymbol{u})+\sqrt{\mu} \sqrt{\left(\frac{\rho\left\|\boldsymbol{a}_{h}\right\| h}{\mu}\right)} \varepsilon_{1}(\boldsymbol{u})+\frac{1}{\sqrt{\mu}} \varepsilon_{0}(\boldsymbol{\sigma})+\frac{1}{\sqrt{\mu}}\left(\frac{\lambda\left\|\boldsymbol{a}_{h}\right\|}{h}\right) \varepsilon_{0}(\boldsymbol{\sigma})+\frac{1}{\sqrt{\mu}} C \varepsilon_{0}(p)
$$

Note that this error function is defined in terms of the local Reynolds number and the local Weissenberg number.

Lemma 1 (consistency). Let $\boldsymbol{U} \in \mathcal{X}$ be the solution of the continuous problem and $\boldsymbol{U}_{h} \in \mathcal{X}_{h}$ the finite element solution. If $\boldsymbol{f} \in \mathcal{V}_{h}$ and $\boldsymbol{U}$ is regular enough, so that $B_{\text {stab }}\left(\boldsymbol{U}, \boldsymbol{V}_{h}\right)$ is well defined, then

$$
B_{\text {stab }}\left(\boldsymbol{U}-\boldsymbol{U}_{h}, \boldsymbol{V}_{h}\right) \leq C E(h)\left\|\boldsymbol{V}_{h}\right\|_{W}
$$


for all $\boldsymbol{V}_{h} \in \mathcal{X}_{h}$.

That $E(h)$ is the error function of the formulation is determined by the following result:

Lemma 2 (interpolation error). Let $\boldsymbol{U} \in \mathcal{X}$ be the solution of the continuous problem, assumed to be regular enough, and $\tilde{\boldsymbol{U}}_{h} \in \mathcal{X}_{h}$ its best finite element approximation. Then, the following inequalities hold:

$$
B_{\text {stab }}\left(\boldsymbol{U}-\tilde{\boldsymbol{U}}_{h}, \boldsymbol{V}_{h}\right) \leq C E(h)\left\|\boldsymbol{V}_{h}\right\|_{W}, \quad\left\|\boldsymbol{U}-\tilde{\boldsymbol{U}}_{h}\right\|_{W} \leq C E(h)
$$

for all $\boldsymbol{V}_{h} \in \mathcal{X}_{h}$

Finally, we can state the main convergence result, which in view of the definition of the error function $E(h)$ turns out to be optimal:

Theorem 2 (convergence). Let $\boldsymbol{U}=(\boldsymbol{u}, p, \boldsymbol{\sigma}) \in \mathcal{X}$ be the solution of the continuous problem. Then there exist a constant $C>0$ such that

$$
\left\|\boldsymbol{U}-\boldsymbol{U}_{h}\right\|_{W} \leq C E(h)
$$

The results stated in terms of the norm $\|\cdot\|_{W}$ are fully meaningful. However, in the case in which viscosity is high and elasticity is small, we can prove stability and convergence in the norms of the spaces where the solution belongs. This is what the following theorem states:

Theorem 3 (stability and convergence in natural norms). The solution of the discrete problem $\boldsymbol{U}_{h}=$ $\left(\boldsymbol{u}_{h}, p_{h}, \boldsymbol{\sigma}_{h}\right) \in \mathcal{X}_{h}$ can be bounded as (for the viscous dominated case and for the low Weissenberg number case):

$$
\sqrt{\mu}\left\|\boldsymbol{u}_{h}\right\|_{H^{1}(\Omega)}+\frac{1}{\sqrt{\mu}}\left\|\boldsymbol{\sigma}_{h}\right\|+\frac{1}{\sqrt{\mu}}\left\|p_{h}\right\| \leq \frac{C}{\sqrt{\mu}}\|\boldsymbol{f}\|_{H^{-1}(\Omega)}
$$

Moreover, if the solution of the continuous problem $\boldsymbol{U}=(\boldsymbol{u}, p, \boldsymbol{\sigma}) \in \mathcal{X}$ is regular enough, the following error estimate holds:

$$
\sqrt{\mu}\left\|\boldsymbol{u}-\boldsymbol{u}_{h}\right\|_{H^{1}(\Omega)}+\frac{1}{\sqrt{\mu}}\left\|\boldsymbol{\sigma}-\boldsymbol{\sigma}_{h}\right\|+\frac{1}{\sqrt{\mu}}\left\|p-p_{h}\right\| \leq C E(h)
$$

Finally, under the classical assumption of elliptic regularity we can prove $L^{2}$ estimates for the velocity:

Theorem 4 ( $L^{2}$-error estimate for the velocity). Suppose that the Reynolds number and the Weissenberg numbers are small, and that the continuous problem satisfies the elliptic regularity condition

$$
\sqrt{\mu}\|\boldsymbol{u}\|_{H^{2}(\Omega)}+\frac{1}{\sqrt{\mu}}\|\boldsymbol{\sigma}\|_{H^{1}(\Omega)}+\frac{1}{\sqrt{\mu}}\|p\|_{H^{1}(\Omega)} \leq \frac{C}{\sqrt{\mu}}\|\boldsymbol{f}\|
$$

Then, there holds:

$$
\sqrt{\mu}\left\|\boldsymbol{u}-\boldsymbol{u}_{h}\right\| \leq C h\left(\sqrt{\mu}\left\|\boldsymbol{u}-\boldsymbol{u}_{h}\right\|_{H^{1}(\Omega)}+\frac{1}{\sqrt{\mu}}\left\|\boldsymbol{\sigma}-\boldsymbol{\sigma}_{h}\right\|+\frac{1}{\sqrt{\mu}}\left\|p-p_{h}\right\|\right)
$$




\begin{tabular}{|c|c|c|c|}
\hline $4: 1$ & \multicolumn{3}{|l|}{ contraction } \\
\hline & $\mathrm{n}^{\circ}$ elements & $\mathrm{n}^{\circ}$ nodes & $\Delta x_{\min }=\Delta y_{\min }$ \\
\hline M1 & 27258 & 27667 & 0.05 \\
\hline M2 & 39894 & 40501 & 0.025 \\
\hline M3 & 105816 & 106671 & 0.01 \\
\hline \multicolumn{4}{|c|}{ over a cylinder } \\
\hline & $\mathrm{n}^{\circ}$ elements & $\mathrm{n}^{\circ}$ nodes & $\Delta x_{\min }=\Delta y_{\min }$ \\
\hline M1 & 41514 & 21330 & 0.025 \\
\hline M2 & 56905 & 29152 & 0.015 \\
\hline M3 & 67856 & 35167 & 0.005 \\
\hline
\end{tabular}

Table 1: Mesh convergence details.

\section{Numerical results}

In this section, some tests are conducted to show the numerical performance of the proposed formulation, emphasizing the robustness of the non-residual one.

The first example corresponds to the $4: 1$ contraction problem, then we show some results of the classical flow over a confined cylinder problem, defined by a pipe of diameter equal to 2 and a cylinder of diameter 1 , and finally, a tridimensional jet-buckling case of a high elastic flow using a two-fluid formulation. In all the cases, the Oldroyd-B constitutive model is used.

In Fig. 1 the spatial discretization is shown for the 4:1 contraction problem and for the cylinder case. In this figure $n_{e}$ represents the number of elements, $n_{n}$ the number of nodes and $\Delta x_{\min }$ represents the minimum element size used in both cases. These mesh sizes were chosen after evaluating the convergence of the results using different meshes for each example. The details of the meshes used to do this are detailed in Table 1. Note that in both cases, the presented results are associated to the M3 mesh but are practically identical to the results of mesh M2.

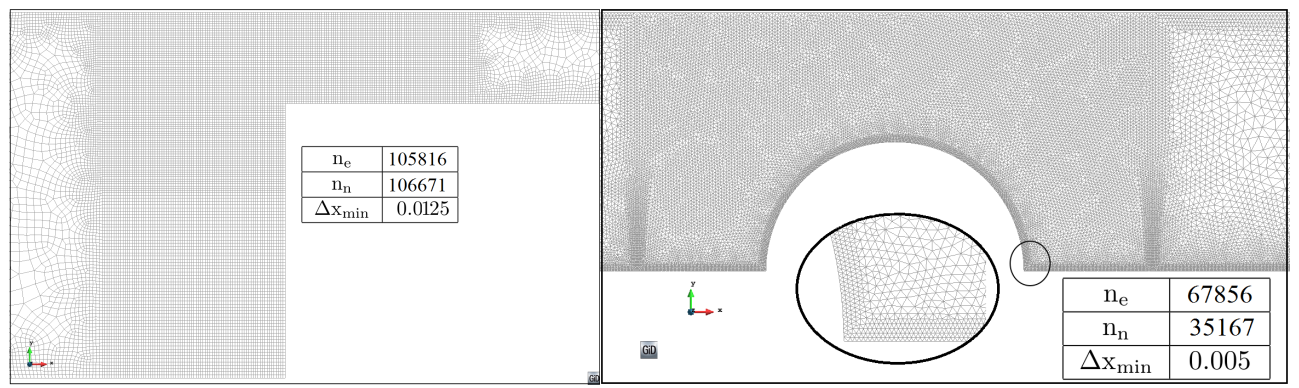

Figure 1: Meshes used: 4:1 contraction (left) and flow over a cylinder (right).

The motivation for the design of the non-residual based formulation is due to the fact that the residual formulation is not robust in the presence of high gradients of the unknowns. Fig. 2 shows the pressure variation along a line near to the corner. The corner is situated in $x=20$. In this figure, the non-residual based and the residual based solutions are shown for a Weissenberg number $\mathrm{We}=2.0$ and a Reynolds number 
$\operatorname{Re}=1.0$. The non-residual formulation captures the peak accurately but the residual one generates a nonphysical secondary peak, that cannot be removed using mesh refinement. From the numerical analysis, the term-by-term formulation permits an orthogonal term-by-term control for the pressure gradient and for the divergence of the elastic stresses, that the residual formulation cannot control, a possible answer for this situation. In any case, it has to be remarked that for smooth solutions, both formulations are stable and display an optimal order of convergence. We have observed that only in cases in which the solution has very high concentrated gradients the non-residual based formulation is more robust, as shown in this example.

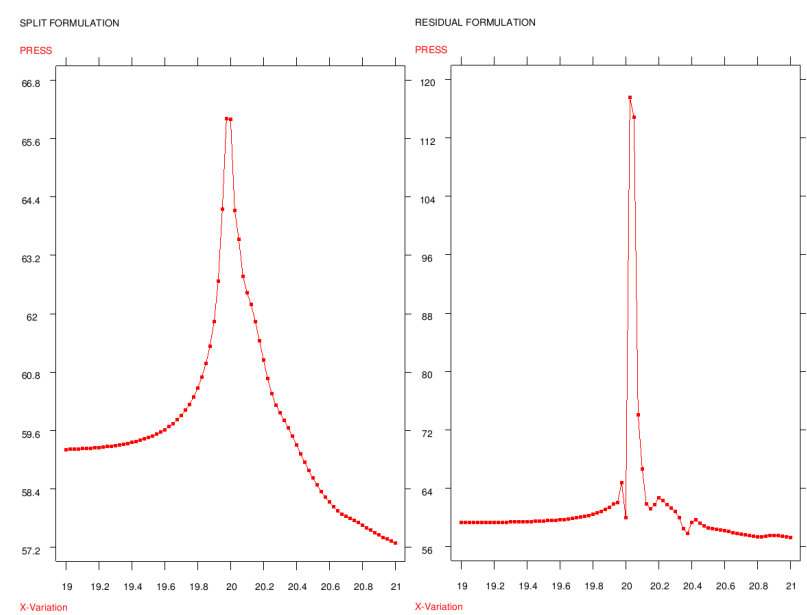

Figure 2: Pressure variation along a line in 4:1 contraction problem (We $=2.0$ and Re $=1.0)$ : non-residual based (left) and residual based (right).

Additionally to a better capability in the resolution of high gradient problems, the non-residual formulation permits the calculation of more elastic flows. In Fig. 3 the streamlines of two cases are shown. Using the residual based formulation, the more elastic case that we have been able to compute is approximately We $=$ 2.0; for more elastic cases the calculations failed to converge. However, the non-residual based formulation is capable to solve the same problem up to $\mathrm{We}=5.0$ (see more results in [15], where the formulation was presented).

In the case of the confined cylinder, where the solution is smooth, the behavior of the non-residual based formulation compared to the residual based one is very similar. In Fig. 4, the drag coefficient obtained with both formulation is compared with published results. Note that in some curves of the graph the word "log" is added, which is associated to the use of the log-conformation approach. In general, what can be found in the literature is that standard formulations can solve the problem for We up to a value between 0.7 and 1.0 depending on the reference $[18,19,5]$, whereas the log-conformation formulation allows one to obtain solutions for We up to a value between 2.0 and 2.5, depending on the source [20,21, 22]. Using our formulations, we have been able to solve the problem up to $\mathrm{We}=1.4$. In this graph, we can see that the agreement is good up to $\mathrm{We}=1.1$, with differences lower than $1 \%$ with respect to the accepted values of 


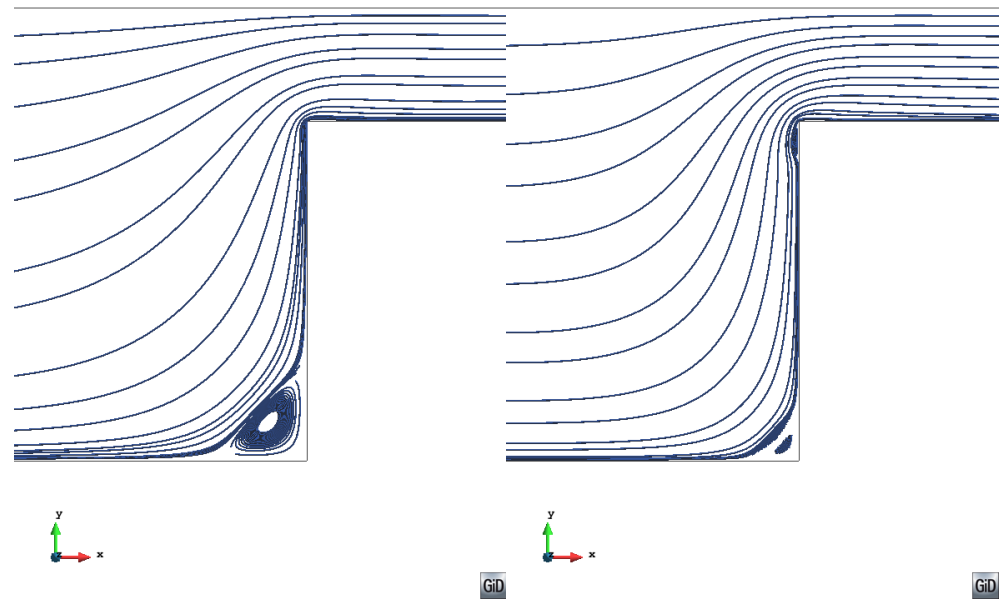

Figure 3: Streamlines in 4:1 the contraction problem: $\mathrm{We}=2.0, \mathrm{Re}=1.0$ (left) and $\mathrm{We}=5.0, \mathrm{Re}=1.0$ (right), both using the non-residual based formulation.

references; for higher values the differences with respect to the log-conformation approach begin to increase, which is possibly associated to the high Weissenberg number problem (HWNP). The term HWNP refers to the empirical observation that all numerical methods break down when the Weissenberg number exceeds a critical value, due to the exponential grown of the stresses.

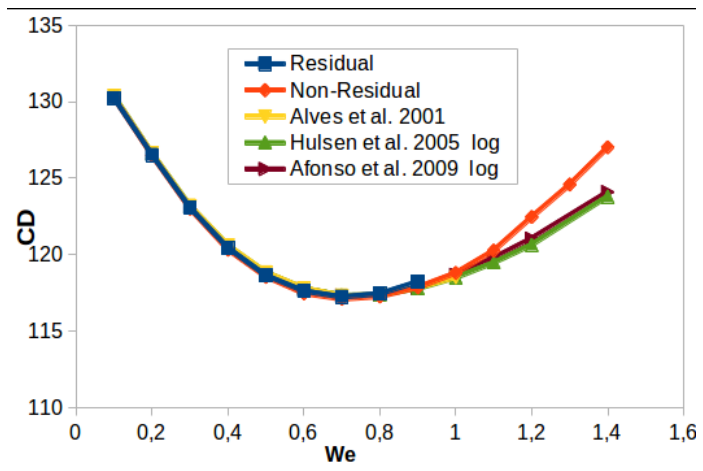

Figure 4: Drag coefficient flow over a cylinder: residual based and non-residual based formulations.

The final numerical example (Fig. 5) is presented to show the robustness of the non-residual based formulation for the three-dimensional case of a jet-buckling flow problem. More details of this example and the two-fluids approach used can be found in [23], from where the example was taken. The only objective to include these results here is to show that the formulation presented is also accurate and robust in situations other than those considered in this paper. 

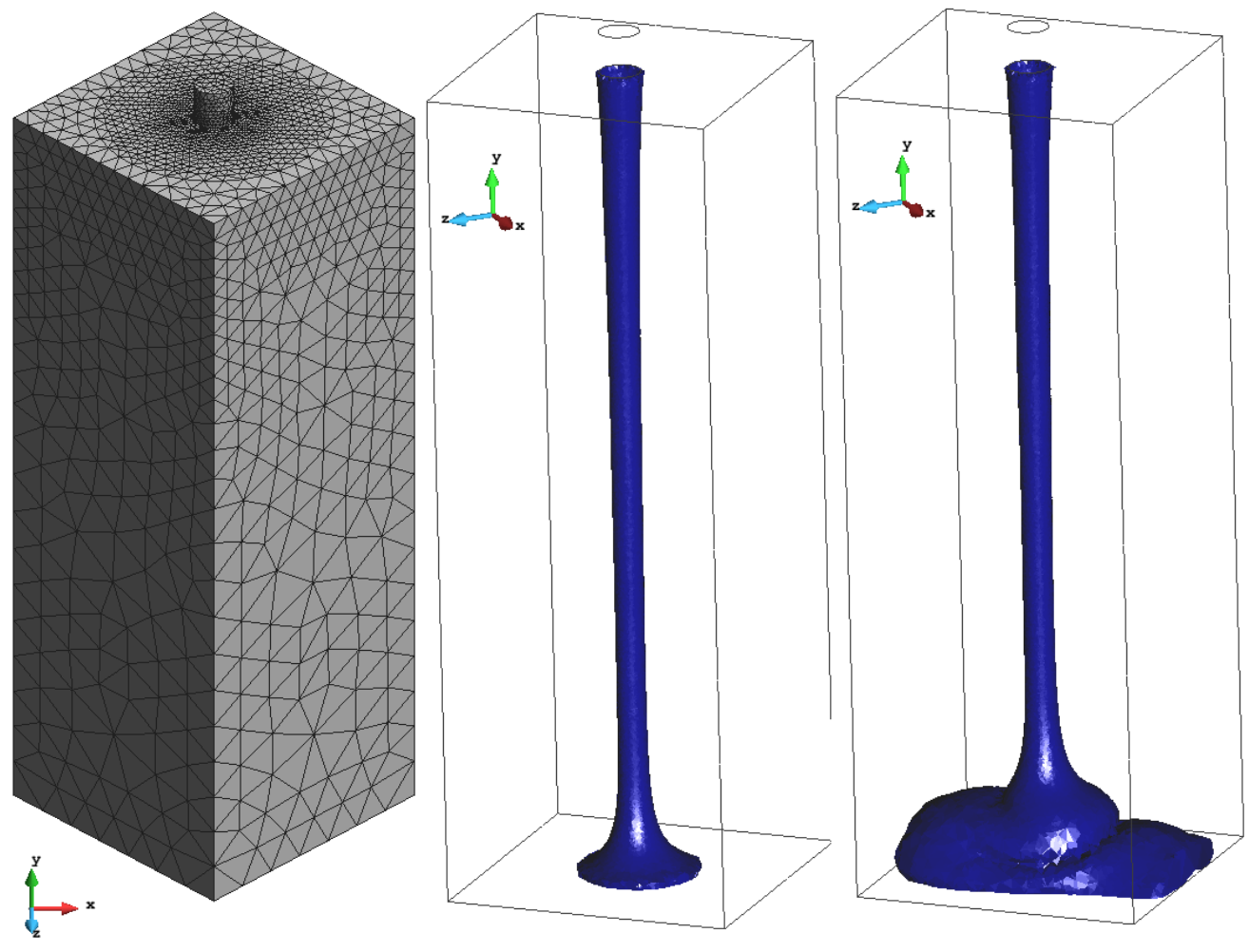

Figure 5: Jet buckling is a $3 \mathrm{D}$ prismatic cavity for: $\mathrm{We}=100$ and $\mathrm{Re}=0.25$. 


\section{Conclusions}

In this paper we have presented a stabilized finite element method to approximate the incompressible viscoelastic flow problem. On the one hand, we have summarized the formulation, stated some results of the numerical analysis of the linearized problem and presented some numerical results. On the other hand, we have explained why the residual based formulation fails in situations in which the stresses have high localized peaks, and how the term-by-term stabilization based on local projections solves the problem. This work can thus be understood as a summary of previous (recent) work and an explanation of the phenomenon described.

The formulation used still suffers from the high Weissenberg number problem. This can also be understood in the light of the estimates presented for the linearized stationary problem, where it is seen that the deteriorate as the Weissenber number (or the Reynolds number) increases. Nevertheless, the present method could also be applied to log-conformation formulations.

Numerical results show the robustness of the method. Once localized oscillations present in residual based formulations have been removed by the term-by-term stabilized method, results obtained are smooth, even for the highest Weissenberg numbers for which results can be found in the literature.

\section{Acknowledgments}

E. Castillo gratefully acknowledges the support received from CONICYT (Programa de Formaciï¡œn de Capital o Humano Avanzado/Becas-Chile Folio 72120080), and from the Universidad de Santiago de Chile. R. Codina gratefully acknowledges the support received from the Catalan Government through the ICREA Acadèmia Research Program.

\section{References}

[1] R. Codina, Finite element approximation of the three-field formulation of the Stokes problem using arbitrary interpolations, SIAM Journal on Numerical Analysis 47 (1) (2009) 699-718.

[2] K. Wang, A new discrete EVSS method for the viscoelastic flows, Computers \& Mathematics with Applications 65 (4) (2013) 609-615.

[3] J. Marchal, M. Crochet, A new mixed finite element for calculating viscoelastic flow, Journal of NonNewtonian Fluid Mechanics 26 (1) (1987) 77-114.

[4] Y. Fan, R. Tanner, N. Phan-Thien, Galerkin/least-square finite-element methods for steady viscoelastic flows, Journal of Non-Newtonian Fluid Mechanics 84 (2-3) (1999) 233-256.

[5] O. M. Coronado, D. Arora, M. Behr, M. Pasquali, Four-field Galerkin/least-squares formulation for viscoelastic fluids, Journal of Non-Newtonian Fluid Mechanics 140 (1-3) (2006) 132-144. 
[6] F. P. Baaijens, Application of low-order Discontinuous Galerkin methods to the analysis of viscoelastic flows, Journal of Non-Newtonian Fluid Mechanics 52 (1) (1994) 37-57.

[7] T. J. Hughes, G. R. Feijóo, L. Mazzei, J.-B. Quincy, The variational multiscale method-a paradigm for computational mechanics, Computer Methods in Applied Mechanics and Engineering 166 (1-2) (1998) 3-24, advances in Stabilized Methods in Computational Mechanics.

[8] R. Codina, Stabilization of incompressibility and convection through orthogonal sub-scales in finite element methods, Computer Methods in Applied Mechanics and Engineering 190 (13-14) (2000) 15791599 .

[9] R. B. Bird, R. C. Amstrong, O. Hassager, in: Dynamics of Polymeric Liquids, vol.1: Fluid Mechanics, 2nd Edition, Wiley, New York, 1987.

[10] R. B. Bird, C. F. Curtiss, R. C. Amstrong, O. Hassager, in: Dynamics of Polymeric Liquids, vol.2: Kinetic Theory, 2nd Edition, Wiley, New York, 1987.

[11] E. Fernández-Cara, F. Guillén, R. Ortega, Mathematical modeling and analysis of viscoelastic fluids of the Oldroyd kind, in Handbook of Numerical Analysis, VIII, North-Holland, 2002.

[12] J. Bonvin, M. Picasso, R. Stenberg, GLS and EVSS methods for a three-field Stokes problem arising from viscoelastic flows, Computer Methods in Applied Mechanics and Engineering 190 (29-30) (2001) 3893-3914.

[13] E. Castillo, R. Codina, Stabilized stress-velocity-pressure finite element formulations of the Navier-Stokes problem for fluids with non-linear viscosity, Computer Methods in Applied Mechanics and Engineering 279 (0) (2014) 554-578.

[14] A. C. Bogaerds, W. M. Verbeeten, G. W. Peters, F. P. Baaijens, 3d viscoelastic analysis of a polymer solution in a complex flow, Computer Methods in Applied Mechanics and Engineering 180 (3-4) (1999) 413-430.

[15] E. Castillo, R. Codina, Variational multi-scale stabilized formulations for the stationary three-field incompressible viscoelastic flow problem, Computer Methods in Applied Mechanics and Engineering 279 (0) (2014) 579-605.

[16] E. Castillo, R. Codina, First, second and third order fractional step methods for the three-field viscoelastic flow problem, Journal of Computational Physics 296 (2015) 113-137.

[17] E. Castillo, R. Codina, Numerical analysis of a stabilized finite element approximation for the three-field linearized viscoelastic fluid problem using arbitrary interpolations, Submitted. 
[18] M. Alves, F. Pinho, P. Oliveira, The flow of viscoelastic fluids past a cylinder: finite-volume highresolution methods, Journal of Non-Newtonian Fluid Mechanics 97 (2-3) (2001) 207-232.

[19] J. Kwack, A. Masud, A three-field formulation for incompressible viscoelastic fluids, International Journal of Engineering Science 48 (11) (2010) 1413-1432, special Issue in Honor of K.R. Rajagopal.

[20] M. A. Hulsen, R. Fattal, R. Kupferman, Flow of viscoelastic fluids past a cylinder at high Weissenberg number: Stabilized simulations using matrix logarithms, Journal of Non-Newtonian Fluid Mechanics 127 (1) (2005) 27-39.

[21] H. Damanik, J. Hron, A. Ouazzi, S. Turek, A monolithic FEM approach for the log-conformation reformulation LCR of viscoelastic flow problems, Journal of Non-Newtonian Fluid Mechanics 165 (1920) (2010) 1105-1113.

[22] A. Afonso, P. Oliveira, F. Pinho, M. Alves, The log-conformation tensor approach in the finite-volume method framework, Journal of Non-Newtonian Fluid Mechanics 157 (1-2) (2009) 55-65.

[23] E. Castillo, J. Baiges, R. Codina, Approximation of the two-fluid flow problem for viscoelastic fluids using the level set method and pressure enriched finite element shape functions, Journal of Non-Newtonian Fluid Mechanics 225 (2015) 37-53. 